\title{
BMJ Open Acupuncture for functional constipation: protocol of an individual patient data meta-analysis
}

Qianhua Zheng, ${ }^{1}$ Hui Zheng, ${ }^{1}$ Lingyun Lu, ${ }^{1}$ Jincheng Leng, ${ }^{1}$ Siyuan Zhou, ${ }^{1}$ Huabin Zheng, ${ }^{2}$ Wenjing Huang, ${ }^{1,3}$ Zhishun Liu, ${ }^{4}$ Bing Zhu, ${ }^{5}$ Ying Li ${ }^{1}$

To cite: Zheng $Q$, Zheng $\mathrm{H}$, Lu L, et al. Acupuncture for functional constipation: protocol of an individual patient data meta-analysis. BMJ Open 2015;5:e007137. doi:10.1136/bmjopen-2014007137

- Prepublication history and additional material is available. To view please visit the journal (http://dx.doi.org/ 10.1136/bmjopen-2014007137).

$\mathrm{ZQH}$ and $\mathrm{ZH}$ contributed equally.

Received 7 November 2014 Revised 4 April 2015 Accepted 12 April 2015

CrossMark

For numbered affiliations see end of article.

Correspondence to

Professor Ying Li;

yingli0213@sina.com

\section{ABSTRACT}

Introduction: Functional constipation (FC) is a common gastrointestinal disease. Systematic reviews indicate that acupuncture may be effective for patients with FC. However, this conclusion is not convincing due to the quality, sample size and methodological heterogeneity of the studies included by these systematic reviews. Therefore, it is necessary for us to conduct a meta-analysis of individual patient data (IPD) from high-quality clinical trials to determine whether acupuncture is effective for patients with FC.

Methods and analysis: Randomised controlled trials (RCTs) of acupuncture for adult patients with FC will be searched in several databases from inception to April 2015. The corresponding authors of eligible studies will be contacted and invited to contribute raw data. The primary outcome is the change in spontaneous defaecation per week from baseline. The secondary outcomes include the proportion of responders, changes in stool quality, mean transit time, proportion of patients using laxatives and adverse events. We will check all of the data and perform reanalysis according to the statistical methodology reported in previous publications. Then we will harmonise the raw data and use a two-step method to conduct the IPD meta-analysis. First, we will calculate the effect size of acupuncture of each trial by analysis of covariance, with the principal end point as the dependent variable and the baseline scores as the covariates. Second, the effect size of acupuncture in each original study will be combined in the metaanalysis.

Dissemination: On the basis of the IPD metaanalysis of high-quality RCTs, this review will answer the question of whether acupuncture is effective for FC. The findings of the review will be disseminated through peer-review publications and conference presentations.

Trial registration number: PROSPERO 2014 CRD42014009901.

\section{INTRODUCTION}

Functional constipation (FG) is a common disease in clinical practice. It includes several symptoms such as decreased frequency of

\section{Strengths and limitations of this study}

- To the best of our knowledge, this will be the first individual patient data meta-analysis to evaluate the effectiveness, efficacy and safety of acupuncture treatment for adult patients with functional constipation. Standardised statistical analysis, adjusted baseline characteristics and subgroups analysis may increase statistical power and reduce heterogeneity.

- Rigorous design of eligible study criteria, methodology of quality assessment, as well as data collection and analysis will guarantee the quality of this study.

- One difficult step of this study is to collect all the raw data of eligible trials by systematic search, which may be the limitation of this meta-analysis. We will actively communicate with the authors and researchers of these trials to ensure that the individual patient data can be collected and synthesised as completely as possible. If not all the raw data can be included, retrieval bias will be analysed in the stage of sensitivity analysis.

defaecation, straining during defaecation, hard stools, sensation of incomplete defaecation and excessive time during defaecation. The diagnosis of FC mainly depends on the individual's symptoms and several physiological examinations. The Rome Foundation published the Rome III criteria in 2006, which listed the consensus criteria for FC. ${ }^{1}$

Several systematic reviews have reported the prevalence of constipation in different populations. It was reported that the prevalence of FC in North America ranged from $12 \%$ to $19 \% .^{2}$ The mean values of prevalence of constipation in the general population of Europe and Oceania were $17.1 \%$ and $15.3 \%$, respectively. ${ }^{3}$ A meta-analysis found that a pooled prevalence of FC in the community was $14 \% .4$ The annual cost for healthcare was $\$ 7522$ for constipation, accounting for $6.5 \%$ of the annual costs for lower gastrointestinal treatment in the USA, reported by Nyrop et $a l^{5}$ Owing to the high incidence 
and the expensive expenditure of FC, patients' quality of life is significantly undermined. Therefore, FC can be considered as a major public health problem. ${ }^{6}$

Treatments for FC vary. Lifestyle and dietary modifications, such as increased physical activity and a fibre-rich diet are widely accepted and recommended by experts as first-line therapy, ${ }^{7}$ although these methods may not be effective for every patient. ${ }^{8}$ Conventional treatments for patients with FC include bulking agents, stool softeners, osmotic and stimulant laxatives, prokinetic agents (tegaserod, cisapride and mosapride) and so forth. ${ }^{9}$ However, the side effects and the expensive expenditures of such treatments are difficult to ignore.

Nowadays, an increasing number of patients with gastrointestinal diseases seek help from complementary and alternative medicine. ${ }^{10}$ Acupuncture, an important part of traditional Chinese medicine (TCM), has been used to treat gastroenterological diseases for a long time, especially functional disorders such as FC, functional diarrhoea and functional dyspepsia. ${ }^{11}$ Many clinical trials, which were conducted to investigate the efficacy of acupuncture for patients with FC, indicated that acupuncture could relieve the patients' symptoms. However, the results of systematic reviews did not reach a definitive conclusion. We found three systematic reviews of acupuncture therapy for patients with FC through systematic search. The first review was published in $2010,{ }^{12}$ which could not draw a conclusion due to the serious methodological flaws of the included studies. The second and third reviews were published in $2012^{13}$ and $2013,{ }^{14}$ respectively. Both of these reviews concluded that acupuncture may have beneficial effects for FC. However, according to the authors' conclusions, the clinical, methodological and statistical heterogeneity of the included studies made the results of these three systematic reviews less convincing.

Since 2012, several new results of randomised controlled trials (RCTs) have been published. It is necessary to update the systematic review using a better method. Therefore, we will perform a meta-analysis based on the individual patient data (IPD) of high-quality RCTs to determine whether acupuncture is effective and safe for people with FC and to calculate the effect size.

\section{METHODS AND ANALYSIS Objectives}

To establish the individual patient database by combining the raw data from high-quality clinical trials of acupuncture treatment for FC, and to answer the question: is acupuncture effective for patients with FC?

We will answer this question in the following aspects:

1. Is acupuncture as effective as the positive drugs?

2. Is real acupuncture superior to sham acupuncture for patients with FC?

3. Is acupuncture superior to no treatment for patients with FC?

4. Is acupuncture safe for patients with FC?

\section{Search strategy}

We will conduct a systematic search of the following databases: MEDLINE, EMBASE, Cochrane Library, Chinese BioMedical Literature Database (CBM), Chinese National Knowledge Infrastructure (CNKI), Chinese Science and Technology Periodical Database (VIP), China's Important Conference Papers Database and China's Dissertation Database from inception to April 2015.

The following search terms will be used individually or combined: "acupuncture," "acupuncture therapy," "auricular acupuncture," "moxibustion," "acupressure," "constipation," "functional constipation," "idiopathic constipation," "randomized/randomised controlled trial," etc. Terms in Chinese will be used in Chinese databases. The search strategy is available in online supplementary additional file 1 . We will also conduct a literature search through the WHO International Clinical Trial Registry Platform (ICTRP) portal and Clinical Trial Registry of the US National Institutes of Health for ongoing registered clinical trials and unpublished articles. Data of ongoing studies will be included after the relevant articles are published in peer-review journals.

\section{Criteria for study eligibility}

Studies will be included in the individual patient database if they meet the following criteria.

\section{Type of studies}

We will include RCTs of acupuncture for FC only. Randomised and allocation concealment methods should be clearly described; if not, we will contact the corresponding authors for further information about the randomisation process. Moreover, allocation concealment should be adequate to avoid selection bias. Additionally, considering that the washout duration of acupuncture cannot be accurately evaluated, we will not include RCTs with crossover designs. To guarantee the quality of an included study, only sample sizes of more than 30 will be considered. The authors of the studies that meet the inclusion criteria will be invited to share the raw data.

\section{Type of participants}

Adult patients who are diagnosed with FC according to the Rome II/III criteria will be included. Patients who are diagnosed with FC (or chronic constipation, primary constipation, idiopathic constipation) with other criteria and who are excluded by examinations for pathological diseases, such as postsurgery, tumour or obstruction, will also be included in this study.

\section{Types of intervention/exposure}

Patients who received acupuncture treatment as the primary intervention will be included, which means that patients in the treatment group of each study were treated by acupuncture or that acupuncture was the main intervention combined with other therapy, such as Chinese herbs or physical exercise. Any type of 
acupuncture will be included, such as manual acupuncture, electroacupuncture, warming-needle moxibustion, auricular acupuncture, scalp acupuncture, pyonex, intradermal needling, acupoint injection with medicine and so forth. According to the Rome II/III criteria, FC is not an acute disease. To alleviate the symptoms, several sessions of acupuncture treatment are necessary in clinical practice. One session of acupuncture treatment only provides acute relief. According to our previous study, the number of treatment sessions in RCTs ranged from $5^{15}$ to $28,{ }^{16}$ and 10 to 20 sessions are most commonly used. Therefore, only RCTs with a treatment protocol of at least 10 sessions will be included.

\section{Types of controls}

At least one control group of patients in the study should receive one of the following interventions: positive drugs, placebo controls or no treatment.

Positive drugs: positive drugs that are used to treat constipation include bulk-forming laxatives, emollients, lubricants, osmotic laxatives, stimulants and chloridechannel activators. ${ }^{17} 18$

Placebo controls include sham acupuncture, placebo drugs, sham interventions and so forth. Sham acupuncture is used to make patients believe they receive acupuncture stimulation, without knowing whether it is real or not. Sham acupuncture includes superficial insertion with needles at specific acupoints; needles puncturing at non-specific acupoints, distal acupoints or non-acupoints; placebo needles, such as Streitberger needles; ${ }^{19}$ and other techniques, which would make patients feel like needle penetration.

No treatment is defined as any of the following: waiting list, which means that patients in the control group do not receive any acupuncture treatment until the trial is completed; general care or usual care, which means that patients in the control group only receive advice and/or health education, such as diet and/or exercise recommendations.

\section{Types of outcome measurements \\ Primary outcomes}

The primary outcome of this review is the change in spontaneous defaecation per week from baseline.

\section{Secondary outcomes}

The secondary outcomes include the following items: proportion of responders, which is the number of responders divided by the total number of participants in each group; changes in stool quality, which are assessed by the Bristol stool scale or other objective measurements; mean transit time, which is the time from the first perception of wanting to defaecate to the finish of defaecation; proportion of patients using laxatives, which is the number of patients who used laxatives during the trial to alleviate their symptoms. In addition, the proportion of adverse events will also be calculated according to patient reports in each study.

\section{Data collection}

\section{Study selection and data extraction}

After electronic searches in the databases, two reviewers will independently screen the titles and abstracts to exclude: (1) the duplicates; (2) the studies in which the participants did not meet the criteria of FC; (3) the studies that were not RCTs with parallel design; and (4) the studies in which the participants in the experimental group did not receive acupuncture treatment as the primary intervention. Then our reviewers will screen the full copies of studies that cannot be clearly screened by titles and abstracts only (the full copies screening form is available in online supplementary additional file 2). Any disagreement will be resolved by consensus or judged by a third reviewer.

Next, two reviewers will independently extract the data from the studies. Training in data extraction and reviewer examination will be conducted in advance to guarantee the quality of extraction. Reviewers will document the following information on the Data Extraction Form (see online supplementary additional file 2): (1) the basic information of the study (the date, reviewer name of data extraction and study details, such as first author, year of publication, country of publication and publication type); (2) the study characteristics (design of the study, sample size, number of groups, methodology of randomisation and allocation concealment, blinding, settings); (3) participants (age, gender, ethnicity, diagnosis, etc); (4) interventions and controls (type of interventions, number and frequency of sessions, duration of treatment or follow-up, etc); (5) outcomes (type of outcome, definition of outcome, time point of assessment, etc); and (6) results (the statistic description of outcomes, such as mean, SD, observed and total sample size, adverse events, etc). Rechecking and discussion, or even the judgement from a third reviewer will be required if there is any disagreement during the data extraction.

\section{Methodological quality assessment}

The assessment of methodological quality is of great importance to systematic reviews. After data extraction, two reviewers will evaluate the methodological quality of each original study independently and document the details in the Risk of bias assessment form. Discrepancies will be resolved by consensus or judged by a third reviewer. According to the Cochrane Collaboration's tool for assessing risk of bias (Cochrane Handbook (V.5.1.0)), the methodological quality will be assessed by the following criteria: (1) randomisation allocation; (2) randomisation concealment; (3) blinding; (4) data integrity; (5) selective reporting; and (6) other bias, such as trial design, baseline similarity of groups, early cessation of treatment and so forth. For all of the studies, the assessment should follow the above six criteria and be categorised as A, B or C grade of risk (low, unclear and high risk of bias, respectively). The risk grade will be used to evaluate the quality of all the studies based on the Cochrane collaboration's recommendation. 
Regarding the characteristics of acupuncture clinical trials, we will also assess the quality of acupuncture interventions according to the Standards for Reporting Interventions in Controlled Trials of Acupuncture (STRICTA) recommendation. ${ }^{20}$ However, it is difficult to achieve blinding for acupuncture treatment. Therefore, we only ask for blinding assessment and statistical analysis. Any disagreements will be resolved by consensus; otherwise, a third reviewer will be consulted.

\section{Raw data collection and checking}

The corresponding authors of eligible studies will be contacted and invited to contribute the raw data. If the authors of the older studies cannot provide the original data, we will present the details in the final report, and further analysis will be necessary in the sensitivity analysis. The raw data will be provided in any manner that is convenient for authors (such as by email) in any type of electronic format, such as SPSS, STATA, R, Excel, etc. After checking the availability of the data files, all of the data will be converted to a uniform format with its own name, which will be composed of the first author's name and the year of publication.

Reviewers will check all the data carefully to determine whether there are any obvious errors, irrational or missing data; if any of these issues are found, we will contact the authors for further information. After checking the data, we will calculate the data according to the statistical methodology of the published papers. If there are any inconsistencies regarding the published results, we will require more information from the investigators. For the studies that are still being conducted, we will ask the investigators to provide the most recent data. Then we will create a new data set for all the raw data and harmonise the names and format of variables, which will be used for further IPD meta-analysis.

For missing data, we will ask the investigators to recheck the missing data and determine whether this omission happened during data entry. If not, we will use multiple imputation by the propensity score methods.

\section{Raw data management}

All the data will be stored in a computer with password protection at the College of Acupuncture and Tuina, Chengdu University of TCM. Only authorised members of this IPD study will be allowed to access the computer. Furthermore, there will be a Data Management Committee to supervise the reasonable and confidential use of the original data. Data governance is intended to guarantee the safety of the relevant data from each study and the interests of multiple parties. We will sign a confidentiality agreement with each raw data provider to ensure the anonymity of the individual patients' data.

\section{Statistical methods}

Heterogeneity

A heterogeneity test is an important step in conducting a meta-analysis, as it determines whether the data from studies are suitable and meaningful to synthesise. The result of a meta-analysis will be valid and reliable if a heterogeneity test is conducted and a reasonable explanation is provided. However, there is still controversy regarding the investigation of heterogeneity in an IPD meta-analysis; thus, we will use a conventional method, $\mathrm{I}^{2}$ value (tested by Higgins I-squared test), to detect the heterogeneity of eligible studies. According to the Handbook of Cochrane, $\mathrm{I}^{2}$ values are divided into four categories, and a value $>50 \%$ will be considered as a significant heterogeneity among the included studies. As previously mentioned, the heterogeneity was significant in the previous systematic reviews of acupuncture for patients with FC. The sources of heterogeneity that we speculate on are differences in patients' demographics, different styles of acupuncture treatment (such as manual acupuncture, electroacupuncture or auricular acupuncture, etc), the alliance of different interventions, different outcomes and time points. Using IPD is an effective way to address these heterogeneity issues. Therefore, there are more possibilities to obtain quantitative results rather than qualitative results. In this study, we will first calculate the effect size of acupuncture of each study by the standardised principle and then conduct a heterogeneity test and meta-analysis. If significant heterogeneity remains, we will perform a metaregression analysis to determine the sources and take appropriate action to address them. An explicit explanation and relevant sensitivity analysis will be available in our final report.

\section{Primary end point}

The primary end point plays a vital role in the final result. We will first identify the primary end point (including the outcome and time point) of each study. In this meta-analysis, we will use the change in spontaneous defaecation frequency per week from baseline, which is a continuous variable. If the outcomes are ordinal data or count data based on times of defaecation, we will convert them to continuous variables. Some studies use Cleveland Clinic Score (CCS) ${ }^{21}$ or colonic transit time $(\mathrm{CTT})^{22}$ after treatment as the primary end point. In this situation, we will create a standardised primary end point divided by pooled SD.

\section{Data analysis}

\section{Primary analysis}

The effect size of acupuncture will be analysed in two steps. First, each original study will be reanalysed by analysis of covariance with the standardised principal end point as the dependent variable and the baseline characteristics as covariates (such as baseline situation, participants' characteristics, etc) to calculate the effect size of acupuncture for FC. Second, all the effect sizes from the original studies will be included in the meta-analysis by the meta or metafor package in $\mathrm{R}$ project (http://www.r-project.org). During this process, we will choose an appropriate effects model, such as the fixed 
effects model or random effects model according to the heterogeneity. If the heterogeneity is not obvious, for example, mild or moderate, we will perform both fixed and random effects models to calculate the effect size and compare the differences. In this step of the analysis, we will detect the effect size of acupuncture separately by comparing acupuncture with positive drugs, placebo controls and no treatment.

\section{Secondary analysis}

We will reanalyse the effect size for the changes in stool quality assessed by the Bristol stool scale or other objective measurements, the proportion of responders, the mean transit time, the proportion of patients using laxatives, and the proportion of adverse events during the studies. Standardised mean differences will be used in the meta-analysis if there are different measurement scales in the original studies. Continuous data will be described as the mean and standardised difference (SD), and categorical data will be described as counts or percentages. Missing values will be addressed by multiple imputation with the propensity score method.

Time points are difficult to determine because of the different study designs. According to the different observation courses, for our data, 4 weeks will be the cut-off point. Before the cut-off point, the time points before 4 weeks will be regarded as the short-term effects, and the points thereafter will be regarded as the long-term effects. We will harmonise the time variables (eg, 1 month is equivalent to 4 weeks). Any time point that investigators designed for assessment close to our uniformed time point will be considered as the time point in this IPD study.

Furthermore, considering some factors that may influence clinical outcomes, we will analyse the characteristics of patients with FC (such as the age, gender, ethnicity and course of disease) as well as the characteristics of acupuncture treatment (such as the number, frequency and duration of treatment sessions, the number of acupoints and the different types of acupuncture interventions) using a logistic regression method. We will determine whether these characteristics influence the effect of acupuncture for FC.

\section{Sensitivity analysis}

Sensitivity analysis will include the following aspects:

First, sensitivity analysis will be performed for publication bias. Although the inclusion and exclusion criteria that we designed can decrease publication bias, we will also perform sensitivity analysis to detect it. A funnel plot will be used if more than 10 studies are included in this IPD study. Furthermore, the fail-safe number will be calculated to determine the degree of bias.

Second, since exclusion and dropouts will be processed by multiple imputation in the statistical analysis according to the available data, a sensitivity analysis will be conducted.
Third, the omitted studies and subgroups will be analysed by sensitivity analysis. According to the quality assessment, some studies with a high risk of bias or a subgroup with a small sample size will not be included in the meta-analysis. However, we will analyse all the data in sensitivity analysis by synthesising these omitted studies or subgroups and compare the results before and after data synthesis.

Fourth, it is known that one difficult step of conducting the IPD meta-analysis is collecting all the raw data from the eligible studies. Although we will actively communicate with the authors and researchers to collect the raw data as completely as possible, there is still a possibility that the raw data will be incomplete. If so, we will first conduct the conventional meta-analysis on the published data of all the eligible studies and of the studies with raw data available separately. Then we will compare the analysis results of these two, in order to identify the possible selection bias.

Finally, as we mentioned above, we will perform both fixed and random effects models to detect heterogeneity during the meta-analysis. Omitted studies that contribute to greater heterogeneity will also be included in the sensitivity analysis.

\section{Ethics and dissemination}

Since each eligible study was approved by local institutional review boards and ethical committees before the trial was conducted, and all of the participants included were required to sign the written informed consent, this IPD meta-analysis study does not require further ethical approval.

This protocol of IPD meta-analysis of acupuncture for FC has been registered with the International Prospective Register of Systematic Reviews (PROSPERO) at the National Health Service Centre for Reviews and Dissemination at the University of York (Registration number: CRD42014009901). All the items recommended by the Preferred Reporting Items for Systematic Review and Meta-analysis Protocols (PRISMA-P) ${ }^{23}$ have been included in this protocol paper. The result of this review will provide valid and reliable evidence of acupuncture treatment for patients with FC. The findings of this review will also provide implications for clinical practice and further research and be disseminated by peerreview publications and conference presentations.

\section{DISCUSSION}

Individual patient data meta-analysis of high-quality trials will provide the most reliable evidence for clinical treatment decisions. On the basis of the solid foundation in both clinical practice and academic research of our research team, we believe that the results of this study will be fruitful and valid to determine definitive conclusions regarding acupuncture treatment for FC.

We designed this IPD meta-analysis protocol based on previous relevant studies. ${ }^{24} 25$ However, our study has 
some limitations. The characteristics of both acupuncture and patients can possibly influence the therapeutic effects of acupuncture in clinical practice. The changes in acupuncture effects over time play a role in creating a treatment schedule. The factors discussed above will be included in this protocol, but we plan to analyse all of the relevant factors in the future to offer more information for clinical practice and scientific research. Moreover, one difficult step of this study is acquiring the complete raw data from original trials. To address this problem, we will take the following steps: first, we will follow the strict inclusion criteria and conduct the systematic search to locate the eligible trials. Second, we will actively contact and communicate with the authors and researchers of included RCTs and invite them to participate in this study and to offer data as completely as possible. Finally, we will strictly follow relevant agreements and contracts signed with every data provider to protect mutual interests during this study. If the raw data cannot be fully obtained, we will report the details and the results of possible bias.

We sincerely hope that the findings of this review will guide future treatments for FC and translate the contributions of clinical research into patient benefits.

\section{Author affiliations}

${ }^{1}$ Chengdu University of Traditional Chinese Medicine, Chengdu, Sichuan, People's Republic of China

${ }^{2}$ First Affiliated Hospital of Chengdu University of Traditional Chinese

Medicine, Chengdu, Sichuan, People's Republic of China

${ }^{3}$ Institute for Social Medicine, Epidemiology, and Health Economics, Charité, University Medical Center, Berlin, Germany

${ }^{4}$ China Academy of Chinese Medical Sciences, Guang'anmen Hospital, Beijing, People's Republic of China

${ }^{5}$ China Academy of Chinese Medical Sciences, Beijing, People's Republic of China

Acknowledgements The authors gratefully acknowledge Dr Xi Wu from Chengdu University of Traditional Chinese Medicine for her advice on the search strategy. The authors thank America Journal Experts (AJE), Jintao Li and Daimin Fei for English language editing.

Contributors The study was conceived and designed by $\mathrm{QHZ}$ and $\mathrm{HZ}$. WJH contributed to the design of statistical analysis and will monitor the quality of the included RCTs. QHZ, HZ, SYZ, LYL, HBZ and JCL form the Data Management Committee and have responsibility for study research and data safety. LYL, JCL and SYZ were involved in collecting, checking and reviewing the data. $\mathrm{QHZ}$ and $\mathrm{HZ}$ are responsible for overall data analysis. $\mathrm{HZ}, \mathrm{YL}, \mathrm{ZSL}$ and BZ, who formed the Research Steering Committee, also contributed suggestions to the design of this study and individual patient data to meta-analysis. YL is responsible for the overall quality of the study. This manuscript was written and revised by $\mathrm{QHZ}$ and $\mathrm{HZ}$. All authors read this manuscript and approved the publication of this protocol.

Funding This study is supported and financed by grants from the National Basic Research Programme (No. 2011CB505205) and the Nation Natural Science Foundation of China (No. 81273853).

Competing interests None declared.

Provenance and peer review Not commissioned; externally peer reviewed.

Data sharing statement This article is only a study protocol of an individual patient data meta-analysis for acupuncture treatment for patients with functional constipation. The results of this study will offer reliable and valid evidence for clinical practice and also be available for further researches. At present, this article does not include relevant data.
Open Access This is an Open Access article distributed in accordance with the Creative Commons Attribution Non Commercial (CC BY-NC 4.0) license, which permits others to distribute, remix, adapt, build upon this work noncommercially, and license their derivative works on different terms, provided the original work is properly cited and the use is non-commercial. See: http:// creativecommons.org/licenses/by-nc/4.0/

\section{REFERENCES}

1. Longstreth GF, Thompson WG, Chey WD, et al. Functional bowel disorders. Gastroenterology 2006;130:1480-91.

2. Higgins PD, Johanson JF. Epidemiology of constipation in North America: a systematic review. Am J Gastroenterol 2004;99:750-9.

3. Peppas G, Alexiou VG, Mourtzoukou E, et al. Epidemiology of constipation in Europe and Oceania: a systematic review. BMC Gastroenterol 2008;8:5.

4. Suares NC, Ford AC. Prevalence of, and risk factors for, chronic idiopathic constipation in the community: systematic review and meta-analysis. Am J Gastroenterol 2011;106:1582-91.

5. Nyrop KA, Palsson OS, Levy RL, et al. Costs of health care for irritable bowel syndrome, chronic constipation, functional diarrhea and functional abdominal pain. Aliment Pharmacol Ther 2007;26:237-48.

6. Wald A, Scarpignato C, Kamm MA, et al. The burden of constipation on quality of life: results of a multinational survey. Aliment Pharmacol Ther 2007;26:227-36.

7. Camilleri M, Bharucha AE. Behavioural and new pharmacological treatment for constipation: getting the balance right. Gut 2010;59:1288-96.

8. Meshkinpour $\mathrm{H}$, Selod S, Movahedi $\mathrm{H}$, et al. Effects of regular exercise in management of chronic idiopathic constipation. Dig Dis Sci 1998;43:2379-83.

9. Liu LW. Chronic constipation: current treatment options. Can J Gastroenterol 2011;25(Suppl B):22B-8.

10. Tillisch K. Complementary and alternative medicine for gastrointestinal disorders. Clin Med 2007;7:224-7.

11. Ouyang H, Chen JD. Review article: therapeutic roles of acupuncture in functional gastrointestinal disorders. Aliment Pharmacol Ther 2004;20:831-41.

12. Li MK, Lee TF, Suen KP. A review on the complementary effects of auriculotherpy in managing constipation. J Altern Complement Med 2010;16:435-47.

13. Du WF, Yu L, Yan XK, et al. Meta-analysis on randomized controlled clinical trials of acupuncture and moxibustion on constipation. Zhongguo Zhen Jiu 2012;32:92-6.

14. Zhang T, Chon TY, Liu B, et al. Efficacy of acupuncture for chronic constipation: a systematic review. Am J Chin Med 2013;41:717-42.

15. Xia CF, Huang LP, Liu GQ. Acupuncture at back-shu acupoints for 45 patients with senile functional constipation. Shanxi Zhongyi 2007;27:95-6.

16. Zhang ZL, Ji XQ, Zhao SH, et al. Multi-central randomized controlled trials of electroacupuncture at Zhigou (TE6) for treatment of constipation induced by stagnation or deficiency of qi. Zhongguo Zhen Jiu 2007;27:475-8.

17. McKay SL, Fravel M, Scanlon C. Management of constipation. J Gerontol Nurs 2012;38:9-15.

18. Bharucha AE, Pemberton JH, Locke GR III. American Gastroenterological Association technical review on constipation. Gastroenterology 2013;144:218-38.

19. Streitberger K, Kleinhenz J. Introduce a placebo needle into acupuncture research. Lancet 1998;352:364-5.

20. MacPherson $\mathrm{H}$, White A, Cummings $\mathrm{M}$, et al. Standards for reporting interventions in controlled trials of acupuncture: the STRICTA recommendation. Standards for Reporting Interventions in Controlled Trials of Acupuncture. Acupunc Med 2002;20:22-5.

21. American college of gastroenterology chronic constipation task force. An evidence based approach to the management of chronic constipation in North America. Am J Gastroenterol 2005;100 (Suppl1):S1-4.

22. Arhan P, Devroede G, Jehanin B, et al. Segmental colonic transit time. Dis Colon Rectum 1981;24:625-9.

23. Shamseer L, Moher D, Clarke M, et al. Preferred reporting items for systematic review and meta-analysis protocols (PRISMA-P) 2015: elaboration and explanation. BMJ 2014;349:97647.

24. Vickers AJ, Cronin AM, Maschino AC, et al. Individual patient data meta-analysis of acupuncture for chronic pain: protocol of the Acupuncture Trialists' Collaboration. Trials 2010;11:90.

25. Vickers AJ, Cronin AM, Maschino AC, et al. Acupuncture for chronic pain: individual patient data meta-analysis. Arch Intern Med 2012;172:1444-53. 